
\title{
25 Research Soure \\ Natural mineral modulated single atom catalyst for effective water treatment
}

\section{Xiongbo Dong}

China University of Geosciences

\section{Zitong Chen}

China University of Geosciences

Aidong Tang

Central South University

\section{Dionysios Dionysiou}

University of Cincinnati https://orcid.org/0000-0002-6974-9197

Yang Huaming ( $\nabla$ hm.yang@cug.edu.cn )

China University of Geosciences

\section{Article}

Keywords: single atom catalysts (SACs), water treatment, wastewater reclamation.

Posted Date: June 12th, 2021

DOI: https://doi.org/10.21203/rs.3.rs-586491/v1

License: (c) (1) This work is licensed under a Creative Commons Attribution 4.0 International License. Read Full License 


\section{Abstract}

Single atom catalysts (SACs) have been growing as an emerging "hot" topic in environmental remediation. Their performance can be rationally optimized via modulating spatial coordination configuration and porous structure of SACs, which is still challenging. Herein, a novel Si, N co-coordinated cobalt SACs ( $\left.\mathrm{p}-\mathrm{CoSi}_{1} \mathrm{~N}_{3} @ \mathrm{D}\right)$ with 3D freestanding architecture was tailored via employing natural mineral (diatomite) as Si source and porous template. Theoretical calculations and experimental analysis reveal that $\mathrm{Si}$ substitution dramatically decreases electronegativity of $\mathrm{CoN}_{4}$ moieties and thus accelerates interaction and electron transfer between peroxymonosulfate and Co single atom center. Moreover, $\mathrm{p}$ $\mathrm{CoSi}_{1} \mathrm{~N}_{3} @ \mathrm{D}$ inherits hierarchically porous architecture of diatomite, providing more accessible cobalt sites and open diffusion channels for peroxymonosulfate and contaminants in water treatment applications. Thanks to optimal coordination structure and porous architecture, $\mathrm{p}-\mathrm{CoSi}_{1} \mathrm{~N}_{3} @ \mathrm{D}$ can serve as highly active catalyst toward peroxymonosulfate activation, with a turn-over frequency of $299.8 \mathrm{~min}^{-1}$ for bisphenol A degradation, surpassing those of catalysts with transition metal SACs or oxides in disclosed literature. This work provides a novel vision for development of SACs towards wastewater reclamation.

\section{Introduction}

Peroxymonosulfate (PMS)-based advanced oxidation processes (AOPs) have sparked broad interest in environmental remediation because of their in situ generation of oxidizing species, such as hydroxyl radical ( $\left({ }^{\circ} \mathrm{OH}\right)$, sulfate radical $\left(\mathrm{SO}_{4}{ }^{--}\right)$, singlet oxygen $\left({ }^{1} \mathrm{O}_{2}\right)$, and superoxide anion radical $\left(\mathrm{O}_{2}{ }^{-{ }^{-}}\right)^{1-}$ ${ }^{6}$. Although cobalt ion $\left(\mathrm{Co}^{2+}\right)$ has been regarded as the most efficient species for homogeneous PMS activation, the potential environmental toxicity and carcinogenicity of residual $\mathrm{Co}^{2+}$ severely impede its global scale application ${ }^{7}$. Cobalt oxides such as $\mathrm{Co}_{3} \mathrm{O}_{4}$ can address above issues, but the relatively low activation efficiency (turnover frequencies (TOF) per Co atom basis) compared to $\mathrm{Co}^{2+}$ remains challenging ${ }^{7-10}$. In recent years, Co single atom catalysts (SACs), as one of the most emerging kinds of heterogeneous catalysts, possess special electronic structure, utmost Co atom utilization efficiency, and uniformly isolated Co active sites ${ }^{11-17}$. Such unique properties make Co SACs an attractive platform to bridge the gap between homogeneous and heterogeneous PMS activation 1,11,17-20.

The Co isolated single atoms grafted to a carbon basal plane with $\mathrm{CoN}_{\mathrm{x}}$ moieties have been proven as desirable catalysts for PMS activation 11,14,18,20-22. In most cases, the reactive site structure was reported as the planar four-coordinated configuration of $\mathrm{CoN}_{4}$ moieties ${ }^{12,13,23-25}$. Nonetheless, the active Co isolated single atom center surrounded by four coordinated $\mathrm{N}$ atoms can remarkably increase the electronegativity of $\mathrm{CoN}_{4}$ moieties ${ }^{15,26,27}$. The strong electronegativity will directly result in a large reaction Gibbs free energy for the adsorption of negatively charged PMS molecules, which further slows down the PMS activation efficiency. Therefore, it is necessary to rationally adjust the geometric and electronic features of $\mathrm{CoN}_{4}$ moieties for boosting PMS activation performance of Co SACs. Previous 
studies have confirmed that using some other atoms (i.e., P, S, B) with relatively weak electronegativity to substitute the $\mathrm{N}$ atoms in $\mathrm{CoN}_{4}$ moieties could be used for decreasing its electronegativity ${ }^{15,26-30}$. Among various substituted candidates, the electronegativity of $\mathrm{Si}(1.90)$ is much weaker than that of $\mathrm{N}(3.04), \mathrm{P}$ (2.19), S (2.58), and B (2.04) ${ }^{31}$. Modulating single-atom Co sites with Si heteroatom will be the more effective strategy for lowering energy barriers of PMS molecules towards active sites. However, this attractive strategy has been little explored so far for rationally tailoring the geometric and electronic coordination of SACs.

Moreover, the pore structures of catalysts play significant roles in PMS activation via modulating the transport rates of PMS and contaminants towards active sites ${ }^{32,33}$. In fact, a large portion of isolated metal centers on inner surface for SACs is blocked from PMS activation. Although SACs derived from metal-organic frameworks (MOF) or zeolitic imidazole framework (ZIF) possess abundant micropores and small mesopores $(<4 \mathrm{~nm})$, the mass diffusion into their inner surface is highly undesiable ${ }^{34,35}$. On the other hand, the micropores and small mesopores are easily occupied by the intermediates during contaminant degradation, further impeding sustainable and efficient PMS activation. Above issues result in the underutilization of isolated metal centers in SACs derived from MOF or ZIF. Within this context, there is an urgent need to develop elegant synthesis strategies towards SACs with tunable pore structure.

At present, simultaneously adjusting the spatial coordination configuration and pore structure of SACs still remains a grand challenge. Our foregoing investigations and previous reports have confirmed that diatomite, as one of the earth-abundant and low-cost minerals, could serve as a promising template for massive fabrication of carbon-based materials with high specific surface area and tunable pore structure 33, 36-38. On the other hand, the Si of diatomite can be doped into the carbon substrate and act as the silicon source of $\mathrm{SiC}^{39,40}$. Inspired by this, we hypothesize that $\mathrm{Si}$ atom of diatomite could be incorporated into the carbon basal plane of SACs by replacing the $\mathrm{N}$ atoms of $\mathrm{CoN}_{4}$ moieties. Taking these factors into account, it is envisaged that systematically exploring diatomite-based precursors would potentially not only tailor the geometry and electronic structures of $\mathrm{CoN}_{4}$ moieties, but also overcome problems of underutilization of isolated Co single atoms on SACs.

In this work, we report the fabrication of $\mathrm{Si}, \mathrm{N}$ co-coordinated Co SACs (denoted as p-CoSi ${ }_{1} \mathrm{~N}_{3} @ \mathrm{D}$ ) with hierarchically 3D porous architecture by a sacrificial diatomite-template strategy. Experimental results unveiled that $\mathrm{Si}$ atom of diatomite is successfully doped into the $\mathrm{CoN}_{4}$ moieties, and the coordination environment of isolated Co single atom is tuned with one silica atom and three nitrogen atoms (denoted as $\mathrm{CoSi}_{1} \mathrm{~N}_{3}$ ). As demonstrated by density functional theory (DFT) calculations, substituting $\mathrm{N}$ atom with Si atom could optimize the reaction Gibbs free energy by adjusting the electron-withdrawing/donating properties of $\mathrm{CoN}_{4}$ moieties and thus accelerate their interaction with PMS molecules. As a result, $\mathrm{p}$ $\mathrm{CoSi}_{1} \mathrm{~N}_{3} @ \mathrm{D}$ exhibits excellent PMS activation efficiency, with an optimal turnover frequency (TOF) as high as $299.8 \mathrm{~min}^{-1}$ for bisphenol A (BPA) degradation, which is higher than that that in all the previous studies with transition metal single atom or oxides tested under similar conditions (Supplementary Table 
1). This study paves a new way to rationally modulate the spatial coordination configuration and pore structures of SACs.

\section{Results}

Catalyst synthesis and characterization. The schematic illustration of synthesis procedures is depicted in Fig. 1. As shown by X-ray diffraction (XRD) crystallography analysis (Supplementary Fig. 1) and scanning electron microscopy (SEM) image analysis (Supplementary Fig. 2), diatomite-template displays discshaped structure with amorphous silica skeleton, and hierarchically penetrable pores with diameters in the $200-400 \mathrm{~nm}$ range which are radially distributed within the disc-shaped diatom. First, we used porous diatomite as hosts to anchor metal precursor guests by electrostatic attraction interaction. The welldefined spatial distribution of metal precursor on diatomite (Supplementary Fig. 3) was beneficial for the uniform dispersion and exposure of single cobalt sites during polymerization and pyrolysis. The asobtained metal-precursors@diatomite composite was mixed with dopamine (DPA) monomers, which polymerized and evenly arranged around isolated metal-precursors@diatomite composite to form diatomite@metal-precursors@DPA composite. Thereafter, the composite was pyrolyzed at high temperature to embed $\mathrm{Si}, \mathrm{N}$-coordinated metal single atom sites onto diatomite derived hierarchically porous carbon (Supplementary Fig. 4). Subsequently, the sacrificial diatomite-template was etched by hydrofluoric acid (20 wt\%) to collect the Co single atom catalysts embedded in Si, N-doped porous carbon (p-CoSi ${ }_{1} \mathrm{~N}_{3} @ \mathrm{D}$ ). The sample without Si coordination, which denoted as $\mathrm{p}-\mathrm{CoN}_{4}$, was also prepared for comparison through a similar method.

XRD pattern of p-CoSi ${ }_{1} \mathrm{~N}_{3} @ \mathrm{D}$ presents only two broad peaks centered at around $26.0^{\circ}$ and $43^{\circ}$ (Supplementary Fig. 6), which match well with (002) and (100) reflections of graphitic carbon respectively (ICSD No. 617290). The presence of graphitic carbon was further elucidated by Raman spectroscopy (Supplementary Fig. 7). No detectable diffraction peaks assigned to metal phases (pure metal, alloy, and oxide) is observed, confirming the possible formation of Co single atoms in p$\mathrm{CoSi}_{1} \mathrm{~N}_{3} @ \mathrm{D}$. As revealed by SEM (Fig. 2a) images, p-CoSi $\mathrm{N}_{3} @ \mathrm{D}$ preserves the disc-shaped structure and hierarchically penetrable pores of diatomite. Lack of detection of aggregated species on p-CoSi $\mathrm{N}_{3} @ \mathrm{D}$ further suggests the absence of metal nanoparticles or aggregates, in accord with the XRD results. Aberration-corrected high-angle annular dark-field scanning transmission electron microscopy (AC HAADF-STEM) images (Fig. 2b and 2c) show the clearly exclusive bright dots labeled with yellow circles, providing solid proof for the existence of isolated metal single atoms. Interestingly, it is found in the magnified atomic-resolution HAADF-STEM image (Fig. 2d) that many atomic pairs with the distance range from $2.10 \AA$ to $2.50 \AA$ are distributed on the carbon basal plane. The corresponding energy dispersive X-ray (EDX) mappings indicate the homogeneous distribution of $\mathrm{Co}, \mathrm{Si}, \mathrm{N}$ and $\mathrm{C}$ atoms throughout the disc-shaped p-CoSi $\mathrm{N}_{3} @ \mathrm{D}$ (Fig. 2d), demonstrating that the isolated Si atom are successfully doped into the Co SACs. The Co and Si loading of p-CoSi $\mathrm{N}_{3} @ \mathrm{D}$ are determined to be 0.05 wt $\%$ and 1.17 wt\% respectively by inductively coupled plasma mass spectroscopy (ICP-MS, Supplementary Table 2) analysis. It is noted that the BET specific surface area (Supplementary Table 3) 
of $\mathrm{p}-\mathrm{CoSi}_{1} \mathrm{~N}_{3} @ \mathrm{D}$ is up to $465.5 \mathrm{~m}^{2} \mathrm{~g}^{-1}$, much higher than that of SACs synthesized using silica as sacrificial template $\left(312.3 \mathrm{~m}^{2} \mathrm{~g}^{-1}\right)^{41}$. The average pore radius of the mesoporous $\mathrm{p}-\mathrm{CoSi}_{1} \mathrm{~N}_{3} @ \mathrm{D}$ extends to $9.9 \mathrm{~nm}$ (Supplementary Fig. 8). The large specific surface area and hierarchically penetrable pores enhance the exposure of abundant active sites and accelerate the mass transfer and charge impregnation into the inner surface, further increasing utilization efficiency of isolated Co active sites.

X-ray photoelectron spectroscopy (XPS) spectrum survey shows that $\mathrm{Si}, \mathrm{N}$, and $\mathrm{C}$ are the dominant components in p-CoSi ${ }_{1} \mathrm{~N}_{3} @ \mathrm{D}$ (Supplementary Fig. 9). No detectable Co signal could be attributed to the much low Co loading (Supplementary Fig. 10). In comparison to $\mathrm{p}-\mathrm{CoN}_{4}(2.96$ at\%), the relative low $\mathrm{N}$ content in $\mathrm{p}-\mathrm{CoSi}_{1} \mathrm{~N}_{3} @ \mathrm{D}(2.03$ at\%) is caused by the substitution of $\mathrm{N}$ atoms by Si atoms. The $\mathrm{N} 1 \mathrm{~s}$ corelevel spectra of $\mathrm{p}-\mathrm{CoSi}_{1} \mathrm{~N}_{3} @ \mathrm{D}$ is well divided into four peaks, including pyridinic N, Co-N, pyrrolic N, and graphitic $\mathrm{N}$ (Supplementary Fig. 11) ${ }^{42-44}$. The pyrrolic $\mathrm{N}$ in binding energy of $400.0 \mathrm{eV}$ served as the dominant chemical species. Most importantly, different from diatomite, only a weak peak located at 101 $\mathrm{eV}$, which associated to the $\mathrm{Si}-\mathrm{C}$ bond, can be observed in the high-resolution Si $2 \mathrm{p}$ XPS spectra of $\mathrm{p}$ $\mathrm{CoSi}_{1} \mathrm{~N}_{3} @ \mathrm{D}$ (Supplementary Fig. 12) ${ }^{45}$. It is further confirmed that the Si atoms are successfully embedded into the carbon basal plane of Co SACs. This result is well corresponded to the detectable CSi bond in the C 1s core-level spectra of p-CoSi $\mathrm{N}_{3} @ D$ (Supplementary Fig. 13).

The coordination environmental and electronic structure of single Co atoms in p-CoSi $\mathrm{N}_{3} @ \mathrm{D}$ were further elucidated by X-ray absorption near-edge structure (XANES) and Extended X-ray absorption fine structure (EXAFS). The XANES curve of p-CoSi $\mathrm{N}_{3} @ \mathrm{DD}$ (Fig. 3a) shows a white line peak between that of Co foil and $\mathrm{CoO}$, demonstrating that $\mathrm{Co}$ single atoms carried positive charges and their oxidation states are situated between 0 and $+2^{44,46,47}$. The Fourier-transformed $k^{3}$-weighted EXAFS curve of $p$-CoSi ${ }_{1} N_{3} @ D$ displays one prominent peak at $1.31 \AA$, corresponding to Co-N scanning path (Fig. 3b). A shoulder peak at around $1.88 \AA$ is consistent with the prediction of $\mathrm{Co}-\mathrm{Si}$ coordination in $\mathrm{CoSi}_{x} \mathrm{~N}_{4-x}$ models (Supplementary Fig. $14 \mathrm{a}, 15 \mathrm{a}, 16 \mathrm{a}$, and 17a), revealing the successful coordination of $\mathrm{Si}$ atoms in $\mathrm{CoN}_{4}$ moieties. No appreciable Co-Co (located at $2.13 \AA$ ) or Co-O (located at $2.8 \AA$ ) coordination further proves the absence of Co particles or cluster in $\mathrm{p}-\mathrm{CoSi}_{1} \mathrm{~N}_{3} @ \mathrm{D}$. Above results manifest the uniform distribution of isolated single Co atoms in p-CoSi ${ }_{1} \mathrm{~N}_{3} @ \mathrm{D}$. Moreover, the EXAFS wavelet transform (WT) analysis was employed to discriminate the atomic configuration of $\mathrm{p}-\mathrm{CoSi}_{1} \mathrm{~N}_{3} @ \mathrm{D}$. The WT contour plots in Co foil and $\mathrm{Co}_{3} \mathrm{O}_{4}$ shows the intensity maxima at $7.1 \AA^{-1}$ and $6.2 \AA^{-1}$ (Fig. $3 \mathrm{C}$ ), corresponding to the Co-Co and Co-O coordination, respectively. By contrast, the WT contour plots in $\mathrm{p}-\mathrm{CoSi}_{1} \mathrm{~N}_{3} @ \mathrm{D}$ exhibits only one intensity maximum at $4.1 \AA^{-1}$ ascribed to Co-N/Si scanning paths, which is consistent with the results in FTEXAFS. Least-squares EXAFS fitting was performed at Co K-edge to extract the quantitative local structure parameters of Co atom in p-CoSi $\mathrm{N}_{3} @ \mathrm{D}$ (Fig. 3d and Supplementary Table 4). The fitting curves demonstrate that the isolated $\mathrm{Co}$ atom is coordinated by one $\mathrm{Si}$ atom and three $\mathrm{N}$ atoms with the atom distance of $2.31 \AA$ (Co-Si coordination) and $1.82 \AA$ ( $\mathrm{Co}-\mathrm{N}$ coordination). Furthermore, the simulated EXAFS spectra were investigated based on the models of $\operatorname{CoN}_{4}, \operatorname{CoSi}_{1} N_{3}, \operatorname{CoSi}_{2} N_{2}$, and $\operatorname{CoSi}_{3} \mathrm{~N}_{1}$ 
(Supplementary Fig.s 14-17). It is shown that the experimental Co K-edge spectrum for $p$-CoSi ${ }_{1} N_{3} @ D$ is in good agreement with the main features of the theoretical EXAFS spectrum for $\operatorname{CoSi}_{1} \mathrm{~N}_{3}$. Based on the EXAFS fittings and simulations, we further confirmed the Si doping into $\mathrm{CoN}_{4}$ moieties and the formation of $\mathrm{CoSi}_{1} \mathrm{~N}_{3}$ moieties in p-CoSi ${ }_{1} \mathrm{~N}_{3} @ D$.

Catalytic activation measurement. The PMS activation performance of catalysts was systematically investigated by monitoring the bisphenol A (BPA) removal. As shown in Fig. 4a, only $5 \%$ of BPA removal could be achieved for $\mathrm{p}-\mathrm{CoN}_{4} / \mathrm{PMS}$ system within $6 \mathrm{~min}$. Surprisingly, more than $99 \%$ of BPA is removed in 5 min using p-CoSi ${ }_{1} \mathrm{~N}_{3} @ D$ as PMS activator. In comparison to the benchmark heterogeneous $\left(\mathrm{Co}^{2+}\right)$ and heterogeneous (commercial $\mathrm{Co}_{3} \mathrm{O}_{4}$ nanoparticles) catalysts, the much faster BPA removal demonstrates that $\mathrm{CoSi}_{1} \mathrm{~N}_{3}$ moieties serve as the major catalytic center for PMS activation (Fig. 4a). To give a clear comparison of the catalytic performance, the turnover frequencies (TOF) per Co atom for BPA removal was measured as well (Supplementary Note 2). The calculated TOF of p-CoSi $\mathrm{N}_{3} @ \mathrm{D}$ (299.8 min $\left.{ }^{1}\right)$ is more than 400,160 and 60 of magnitude compared to that of $\mathrm{p}-\mathrm{CoN}_{4}\left(0.72 \mathrm{~min}^{-1}\right), \mathrm{Co}_{3} \mathrm{O}_{4}\left(1.81 \mathrm{~min}^{-}\right.$ $\left.{ }^{1}\right)$, and $\mathrm{Co}^{2+}\left(4.74 \mathrm{~min}^{-1}\right)$, respectively. It is worthwhile mentioning that the catalytic performance for PMS achieved here surpasses that in all the previous studies with transition metal single atom or oxides tested under similar conditions (Supplementary Table 1). In addition, the p-CoSi $\mathrm{N}_{3} @ \mathrm{D}$ exhibits excellent stability and reusability in PMS activation (Fig. 4b, Supplementary Fig. 18-20, Supplementary Table 5). The p-CoSi ${ }_{1} \mathrm{~N}_{3} @ \mathrm{D} / \mathrm{PMS}$ oxidation system maintains high BPA degradation efficiency with the change of initial $\mathrm{pH}$ values, inorganic anions, and NOM (Supplementary Fig. 21-24). These results confirm that p$\mathrm{CoSi}_{1} \mathrm{~N}_{3} @ \mathrm{D}$ is a promising candidate for environmental recovery via activation of PMS.

Radical $\left(\mathrm{SO}_{4}{ }^{\cdot-}\right.$ and $\left.{ }^{\circ} \mathrm{OH}\right)$ and nonradical $\left({ }^{1} \mathrm{O}_{2}\right)$ reaction processes generally contribute to the pollutant degradation during PMS activation ${ }^{20,48}$. However, which one dominates is still not clear even in the highly similar Co SACs/PMS systems ${ }^{14,20-22,48}$. Herein, the involved reaction mechanisms were discriminated by in situ EPR and scavenging experiments. A set of characteristic signals for DMPO- $\mathrm{SO}_{4}{ }^{-{ }^{-}}$, DMPO$\cdot \mathrm{OH}$, and TEMP- ${ }^{1} \mathrm{O}_{2}$ adducts observed in Fig. $5 \mathrm{a}$ and $5 \mathrm{~b}$ reveal that the radical and nonradical reaction processes might both occur in $\mathrm{p}-\mathrm{CoSi}_{1} \mathrm{~N}_{3} @ \mathrm{D} / \mathrm{PMS}$ system ${ }^{21,22}$. Interestingly, the BPA removal is dramatically suppressed by furfuryl alcohol (FFA, a scavenger for ${ }^{1} \mathrm{O}_{2}$ ), while nearly no inhibition could be detected within the addition of tert-butanol (TBA, a scavenger for ${ }^{\circ} \mathrm{OH}$ ) and methanol (Me, a scavenger for $\mathrm{SO}_{4}{ }^{-{ }^{-}}$and $\left.{ }^{\circ} \mathrm{OH}\right)$ (Fig. $\left.5 \mathrm{c}\right)^{8,33}$. These results demonstrate that only the nonradical reaction process plays significant role in BPA degradation when $\mathrm{p}-\mathrm{CoSi}_{1} \mathrm{~N}_{3} @ \mathrm{D}$ acts as catalyst for PMS activation. Previous studies have proven that atrazine could not be removed by nonradical reaction process ${ }^{49}$. Nevertheless, a high atrazine degradation efficiency is achieved in $\mathrm{p}-\mathrm{CoSi}_{1} \mathrm{~N}_{3} @ \mathrm{D} / \mathrm{PMS}$ system (Supplementary Fig. 25). This fact demonstrates that the radical reaction process dominates the atrazine degradation, further confirming by scavenging experiments (Supplementary Fig. 26). Based on the above results, we conclude that the proceeding through radical or nonradical reaction process mainly 
depend on the type of pollutants in p-CoSi ${ }_{1} \mathrm{~N}_{3} @ \mathrm{D} / \mathrm{PMS}$ system. In this work, the difference between in situ EPR and scavenging result could be ascribed to the selectivity of ${ }^{1} \mathrm{O}_{2}$ for BPA removal.

Catalyst structure during PMS activation. The electron transfer between PMS molecules and Co active centers was regarded as the rate determining process in the nonradical pathway ${ }^{14,18}$. To gain further insights into the relationship between electron transfer, PMS activation properties and spatial coordination configuration of cobalt single atom active sites, first principles density-function theory (DFT) calculations were further carried out. Based on the above characterization and analysis results, we constructed and optimized the interfacial configurations of two possible models ( $\operatorname{CoN}_{4}$ and $\operatorname{CoSi}_{1} \mathrm{~N}_{3}$ ) (Fig. $5 \mathrm{a}$ and $5 \mathrm{~b}$ ). The $\mathrm{Co}$ single atom center of $\mathrm{CoSi}_{1} \mathrm{~N}_{3}$ displays a less positive-charge distribution density than that of $\mathrm{CoN}_{4}$, proving that the introduction of $\mathrm{Si}$ atoms could successfully slow down the electronegativity of $\mathrm{CoN}_{4}$ moieties. The adsorption structure of PMS molecules on $\mathrm{CoN}_{4}$ and $\mathrm{CoSi}_{1} \mathrm{~N}_{3}$ are optimized as depicted in Fig. $5 \mathrm{c}$ and $5 \mathrm{~d}$. When PMS molecules are adsorbed onto the Co single atom center of $\mathrm{CoN}_{4}$ and $\mathrm{CoSi}_{1} \mathrm{~N}_{3}$ moieties, the $0-0$ bond length in free PMS molecule is extended from $1.394 \AA$ to $1.473 \AA$ and $1.474 \AA$, respectively. However, there is no obvious difference in $0-0$ bond length after doping $\mathrm{CoN}_{4}$ moieties with $\mathrm{Si}$ atoms. It is concluded that modulating the geometric and electronic coordination of single atom Co sites with Si plays a negligible role in breaking $0-0$ bond of PMS molecules $^{8}$. In addition, the electron density difference proves the electrons transfer from PMS to Co single atom active center in both $\mathrm{CoN}_{4}$ and $\mathrm{CoSi}_{1} \mathrm{~N}_{3}$ moieties (Fig. $5 \mathrm{e}$ and $\left.5 f\right)^{14}$. The process of electron transfer is further confirmed by the obviously increased cathodic current densities for the p-CoSi $\mathrm{N}_{3} @ D$ electrode with the addition of PMS in the LSV analysis (Supplementary Fig. 27) ${ }^{18,50,51}$. In comparison to $\mathrm{CoN}_{4}$, the $\mathrm{CoSi}_{1} \mathrm{~N}_{3}$ moieties possess a larger electron density difference of PMS adsorbed on the Co single atoms. This phenomenon demonstrates the remarkably enhanced electron transfer efficiency after doping Si atom into the $\mathrm{CoN}_{4}$ moieties, which is consistent with the results of the much higher change of current output in the chronoamperometric curves for $\mathrm{p}-\mathrm{CoSi}_{1} \mathrm{~N}_{3} @ \mathrm{D}$ (compared with p-CoN $\mathrm{N}_{4}$ )

(Supplementary Fig. 28) ${ }^{8,33}$. Moreover, the calculated adsorption energy of PMS on $\operatorname{CoN}_{4}$ and $\operatorname{CoSi}_{1} \mathrm{~N}_{3}$ moieties are $-3.21 \mathrm{eV}$ and $-5.37 \mathrm{eV}$ respectively (Supplementary Table 6), suggesting that PMS adsorption on $\mathrm{CoN}_{4}$ and $\mathrm{CoSi}_{1} \mathrm{~N}_{3}$ is thermodynamically feasible. By contrast, the much lower adsorption energy for $\mathrm{CoSi}_{1} \mathrm{~N}_{3}$ manifests that the $\mathrm{Si}$ and $\mathrm{N}$ dual-coordinated single atom Co sites dramatically reduce the energy barriers, and thus accelerating the interaction and electron transfer between PMS molecules and Co single atom active center. Above optimal electron transfer process caused by the modulation of coordination environment of Co single atom sites further contributes to the much higher PMS activation performance of $\mathrm{p}-\mathrm{CoSi}_{1} \mathrm{~N}_{3} @ \mathrm{D}$.

\section{Discussion}

In summary, we have demonstrated that the PMS activation performance of Co SACs was tailored and optimized via using $\mathrm{Si}$ atom substitution to modulate the geometric and electronic coordination of $\mathrm{CoN}_{4}$ moieties for the first time. DFT analysis and experimental results confirm that each Co single atom in p- 
$\mathrm{CoSi}_{1} \mathrm{~N}_{3} @ \mathrm{D}$ is surrounded by one $\mathrm{Si}$ atom and three $\mathrm{N}$ atoms. Such unique structure with relatively low electronegativity could further decrease the energy barrier and accelerate the electron transfer between PMS and Co single atom active center. Moreover, as-prepared p-CoSi ${ }_{1} \mathrm{~N}_{3} @ \mathrm{D}$ inherits the hierarchically 3D porous and freestanding architecture, and thus possessing abundant accessible reactive sites. Above characteristics endow p-CoSi ${ }_{1} \mathrm{~N}_{3} @ \mathrm{D}$ outstanding PMS activation performance for BPA degradation with the TOF of $299.8 \mathrm{~min}^{-1}$, outperforming all current transition metal single atoms or oxides tested under similar conditions. The discovery in this work not only provides an effective method for tunable design of the spatial coordination configuration of SACs catalysts, but also paves the way for large-scale preparation of hierarchically porous single atom catalysts for industrial applications.

\section{Methods}

Materials. The purified diatomite was prepared via the scrubbing method as reported in a previous study ${ }^{38}$. The elemental composition of purified diatomite was listed in Supplementary Table 7. Dopamine hydrochloride $\left(\mathrm{C}_{8} \mathrm{H}_{11} \mathrm{NO}_{2 \star} \mathrm{HCl}, 98 \%\right)$, Tri (hydroxymethyl) aminomethane hydrochloride (Tris $\mathrm{HCl}$, $\left.\mathrm{C}_{4} \mathrm{H}_{12} \mathrm{CINO}_{3}, 99 \%\right)$, peroxymonosulfate $\left(2 \mathrm{KHSO}_{5} \cdot \mathrm{KHSO}_{4} \cdot \mathrm{K}_{2} \mathrm{SO}_{4}, \mathrm{PMS}\right)$, bisphenol A (BPA), methanol $(\mathrm{MeOH}), \mathrm{p}$-benzoquinone (p-BQ), tert-butyl alcohol (TBA), and furfuryl alcohol (FFA) were purchased from Shanghai Macklin Biochemical Co. Ltd. 5,5-dimethyl-1-pyrroline-N-oxide (DMPO) and 2,2,6,6-tetramethyl4-piperidone (TEMP) were purchased from Sigma-Aldrich Reagent Co. Ltd. Cobalt acetate tetrahydrate $\left(\mathrm{C}_{4} \mathrm{H}_{6} \mathrm{CoO}_{4} \otimes 4 \mathrm{H}_{2} \mathrm{O}, 99.5 \%\right)$, sodium chloride $(\mathrm{NaCl})$, sodium nitrate $\left(\mathrm{NaNO}_{3}\right)$, sodium sulphate $\left(\mathrm{Na}_{2} \mathrm{SO}_{4}\right)$, sodium bicarbonate $\left(\mathrm{NaHCO}_{3}\right)$, sodium dihydrogen phosphate $\left(\mathrm{NaH}_{2} \mathrm{PO}_{4}\right)$, humic acid $(\mathrm{HA})$, hydrochloric acid $(\mathrm{HCl}, 37 \%)$, sodium hydroxide $(\mathrm{NaOH}, 97 \%)$, hydrofluoric acid $(\mathrm{HF}, 40 \mathrm{wt} \%)$, and other chemicals were purchased from China National Pharmaceutical Group Co. Ltd. (Sinopharm). All chemicals were used as received without further purification. Deionized water $(18 \mathrm{M} \Omega \llbracket \mathrm{cm})$ was used throughout the experiments.

Synthesis of NPs-CoN $\mathbf{4} \cdot 10.8 \mathrm{mg}$ of cobalt acetate tetrahydrate was first dissolved in $100 \mathrm{~mL}$ of deionized water, and then $150 \mathrm{mg}$ of dopamine hydrochloride was dissolved in the solution. Afterwards, $1 \mathrm{~mL}$ of Tris-buffer $(1 \mathrm{mM}, \mathrm{pH}=8.5)$ was added into above solution and stirred in ice water bath for $12 \mathrm{~h}$. The suspension was filtered and washed with deionized water for several times, and dried at $60^{\circ} \mathrm{C}$ under vacuum overnight. The resulting black powder was annealed at $900^{\circ} \mathrm{C}$ for $2 \mathrm{~h}$ under $\mathrm{N}_{2}$ atmosphere to yield NPs-CoN . $_{\text {. }}$

Synthesis of $\mathrm{p}-\mathrm{CoN}_{4}$. The metal solids were etched by immersing NPs-CoN ${ }_{4}$ in the HF (20\%) solution for $24 \mathrm{~h}$. The suspension was centrifuged and washed with deionized water several times, and dried at $60^{\circ} \mathrm{C}$ under vacuum overnight to obtain the $\mathrm{p}-\mathrm{CoN}_{4}$.

Synthesis of $\mathrm{CoSi}_{1} \mathrm{~N}_{3} @ \mathrm{D}$. $800 \mathrm{mg}$ of diatomite and $10.8 \mathrm{mg}$ of cobalt acetate tetrahydrate were thoroughly dispersed into $100 \mathrm{~mL}$ of deionized water after 10 min ultrasonication, and then $150 \mathrm{mg}$ of dopamine hydrochloride was fully dissolved in the suspension. Afterwards, $1 \mathrm{~mL}$ of Tris-buffer $(1 \mathrm{mM}, \mathrm{pH}$ $=8.5$ ) was added into above suspension and stirred in ice water bath for $12 \mathrm{~h}$. The suspension was 
filtered and washed with deionized water for several times, and dried at $60^{\circ} \mathrm{C}$ under vacuum overnight. The resulting black powder was annealed at $900{ }^{\circ} \mathrm{C}$ for $2 \mathrm{~h}$ under $\mathrm{N}_{2}$ atmosphere to yield $\mathrm{CoSi}_{1} \mathrm{~N}_{3} @ \mathrm{D}$.

Synthesis of $\mathrm{p}-\mathrm{CoSi}_{1} \mathrm{~N}_{3} @ \mathrm{D}$. The diatomite template and metal solids were etched by immersing p$\mathrm{CoSi}_{1} \mathrm{~N}_{3} @ \mathrm{D}$ in the HF (20\%) solution for 24 h. The suspension was centrifuged and washed with deionized water several times, and dried at $60{ }^{\circ} \mathrm{C}$ under vacuum overnight to obtain the p-CoSi $\mathrm{N}_{3} @ \mathrm{D}$.

Characterization. The crystalline structure and phase purity were characterized by X-ray powder diffraction (XRD, Bruker D8 diffractometer, Cu Ka radiation). The morphologies were collected by field emission scanning electron microscopy (FESEM, JEOL JSM 6700F). The high-resolution HAADF-STEM and energy dispersive spectroscopy (EDS) images were acquired using a double-aberration-corrected FEI Titan3 G2 60-300 instrument operating at $300 \mathrm{kV}$. The transition metal concentrations were evaluated by inductively coupled plasma mass spectrometry (ICP-MS, Agilent 7700). The chemical and electronic states were recorded by X-ray photoelectron spectroscopy (XPS, Thermo Scientific K-Alpha ${ }^{+}$). The BET specific surface area and pore size distribution was calculated using nitrogen absorptiondesorption isotherms at $77 \mathrm{~K}$ (BET, ASAP240). Detection of active radicals were recorded on a Bruker A300-10/12 using DMPO and TEMP as trapping agents. The $X$-ray absorption near-edge structure (XANES) and extended X-ray absorption fine structure (EXAFS) spectra of Co K-edge were measured in a transmission mode at BL14W1 station in Shanghai Synchrotron Radiation Facility. Co foil, $\mathrm{CoO}, \mathrm{Co}_{3} \mathrm{O}_{4}$, and cobalt phthalocyanine (CoPc) were regarded as the standard references.

Catalytic performance measurements. All degradation experiments were conducted in $100 \mathrm{~mL}$ centrifuge tubes. The initial pH was adjusted by $\mathrm{NaOH}(10 \%)$ and $\mathrm{H}_{2} \mathrm{SO}_{4}(10 \%)$. Firstly, a given quantity of catalysts was thoroughly dispersed in $60 \mathrm{~mL}$ of BPA aqueous solution $(20 \mathrm{mg} / \mathrm{L})$. The resultant suspension was rotated for $30 \mathrm{~min}$ until reaching an adsorption/desorption equilibrium between catalysts and BPA. The reaction was initiated with the addition of a certain amount of PMS. $0.5 \mathrm{~mL}$ of samples were collected and mixed with $0.5 \mathrm{~mL}$ of $\mathrm{MeOH}$ (quenching the unreacted PMS) at predetermined time intervals, and immediately filtered through a Millipore filter (pore size $220 \mathrm{~nm}$ ) to remove catalysts. All degradation experiments were performed in triplicate. The evaluation methods of BPA concentration were presented in Supplementary Note 1.

\section{Declarations}

\section{Data availability}

The data that support the findings of this study and plots within this paper are available from the corresponding authors upon reasonable request.

\section{Acknowledgements}

This work was financially supported by the China Postdoctoral Science Foundation (2020TQ0298). 


\section{Author information}

\section{Affiliations}

Engineering Research Center of Nano-Geomaterials of Ministry of Education, China University of Geosciences, Wuhan, China

Xiongbo Dong, Zitong Chen \& Huaming Yang

School of Chemistry and Chemical Engineering, Central South University, Changsha, China

Aidong Tang

Environmental Engineering and Science Program, Department of Chemical and Environmental Engineering, University of Cincinnati, Cincinnati, $\mathrm{OH}$, USA

Dionysios D. Dionysiou

School of Minerals Processing and Bioengineering, Central South University, Changsha, China

Huaming Yang

\section{Contributions}

H. Y. conceived the project. X. D. wrote initial drafts of the work. D. D. and A. T. offered the help in revising the drafts. H. Y. wrote the final paper. X. D designed the experiments, synthesized, and characterized the materials. X. D., H. Y., A. T., and Z. C. analyzed the data. All of the authors contributed to the scientific discussion.

\section{Corresponding authors}

Correspondence to Huaming Yang.

\section{Competing Interests statement}

The authors declare no conflict of interest.

\section{References}

1. Shang, Y., Xu, X., Gao, B., Wang, S., Duan, X. Single-atom catalysis in advanced oxidation processes for environmental remediation. Chemical Society Reviews, 2021. doi.org/10.1039/D0CS01032D

2. Ahn, Y., Choi, J., Kim, M., Kim, M., Lee, D., Bang, W., et al. Chloride-ediated enhancement in heatinduced activation of peroxymonosulfate: New reaction pathways for oxidizing radical production. Environmental Science \& Technology, 2021. doi.org/10.1021/acs.est.0c07964 
3. Kim, E., Park, S., Adil, S., Lee, S., Cho, K. Biogeochemical alteration of an aquifer soil during in situ chemical oxidation by hydrogen peroxide and peroxymonosulfate. Environmental Science \& Technology, 2021. doi.org/10.1021/acs.est.0c06206

4. Li, Z., Wang, L., Liu, Y., He, P., Zhang, X., Chen, J., et al. Overlooked enhancement of chloride ion on the transformation of reactive species in peroxymonosulfate/ $\mathrm{Fe}(\mathrm{II}) / \mathrm{NH}_{2} \mathrm{OH}$ system. Water Research 195, 116973 (2021).

5. Ding, J., Nie, H., Wang, S., Chen, Y., Wan, Y., Wang, J., et al. Transformation of acetaminophen in solution containing both peroxymonosulfate and chlorine: Performance, mechanism, and disinfection byproduct formation. Water Research 189, 116605 (2021).

6. Guo, Z., Li, C., Gao, M., Han, X., Zhang, Y., Zhang, W., et al. Mn-O covalency governs the intrinsic activity of Co-Mn spinel oxides for boosted peroxymonosulfate activation. Angewandte Chemie International Edition 60, 274-280 (2021).

7. Anipsitakis, G., Dionysiou, D., Gonzalez, M. Cobalt-mediated activation of peroxymonosulfate and sulfate radical attack on phenolic compounds. Implications of chloride ions. Environmental Science \& Technology 40, 1000-1007 (2006).

8. Dong, X., Duan, X., Sun, Z., Zhang, X., Li, C., Yang, S., et al. Natural illite-based ultrafine cobalt oxide with abundant oxygen-vacancies for highly efficient Fenton-like catalysis. Applied Catalysis B: Environmental 261, 118214 (2020).

9. Lim, J., Yang, Y., Hoffmann, M. Activation of peroxymonosulfate by oxygen vacancies-enriched cobalt-doped black $\mathrm{TiO}_{2}$ nanotubes for the removal of organic pollutants. Environmental Science \& Technology 53, 6972-6980 (2019).

10. Gao, H., Yang, H., Xu, J., Zhang, S., Li, J. Strongly coupled g- $\mathrm{C}_{3} \mathrm{~N}_{4}$ nanosheets- $\mathrm{Co}_{3} \mathrm{O}_{4}$ quantum dots as 2D/OD heterostructure composite for peroxymonosulfate activation. Small 14, 1801353 (2018).

11. Li, J., Zhao, S., Zhang, L., Jiang, S., Yang, S., Wang, S., et al. Cobalt single atoms embedded in nitrogen-doped graphene for selective oxidation of benzyl alcohol by activated peroxymonosulfate. Small, 2004579 (2021).

12. Wu, F., Pan, C., He, C., Han, Y., Ma, W., Wei, H., et al. Single-atom Co- $\mathrm{N}_{4}$ electrocatalyst enabling fourelectron oxygen reduction with enhanced hydrogen peroxide tolerance for selective sensing. Journal of the American Chemical Society 142, 16861-16867 (2020).

13. Chu, C., Zhu, Q., Pan, Z., Gupta, S., Huang, D., Du, Y., et al. Spatially separating redox centers on 2D carbon nitride with cobalt single atom for photocatalytic $\mathrm{H}_{2} \mathrm{O}_{2}$ production. Proceedings of the National Academy of Sciences 117, 6376-6382 (2020). 
14. Mi, X., Wang, P., Xu, S., Su, L., Zhong, H., Wang, H., et al. Almost $100 \%$ peroxymonosulfate conversion to singlet oxygen on single-atom $\mathrm{CoN}_{2+2}$ sites. Angewandte Chemie International Edition 133, 4638-4643 (2021).

15. Wan, J., Zhao, Z., Shang, H., Peng, B., Chen, W., Pei, J., et al. In situ phosphatizing of triphenylphosphine encapsulated within metal-organic frameworks to design atomic $\mathrm{Co}_{1}-\mathrm{P}_{1} \mathrm{~N}_{3}$ interfacial structure for promoting catalytic performance. Journal of the American Chemical Society 142, 8431-8439 (2020).

16. Peng, X., Liu. $H_{\text {, }}$ Zhang, Y., Huang, Z., Yang, L., Jiang, Y., et al. Highly efficient ammonia synthesis at low temperature over Ru-Co dual single-atom catalyst. Chemical Science, 2021. DOI:

10.1039/D1SC00304F

17. Song, H., Du, R., Wang, Y., Zu, D., Zhou, R., Cai, Y., et al. Anchoring single atom cobalt on twodimensional MXene for activation of peroxymonosulfate. Applied Catalysis B: Environmenta/ 286, 119898 (2021).

18. Li, J., Zhao, S., Yang, S., Wang, S., Sun, H., Jiang, S., et al. Atomically dispersed cobalt on graphitic carbon nitride as a robust catalyst for selective oxidation of ethylbenzene by peroxymonosulfate. Journal of Materials Chemistry A 9, 3029-3035 (2021).

19. Qi, Y., Li, J., Zhang, Y., Cao, Q., Si, Y., Wu, Z., et al. Novel lignin-based single atom catalysts as peroxymonosulfate activator for pollutants degradation: Role of single cobalt and electron transfer pathway. Applied Catalysis B: Environmental 286, 119910 (2021).

20. Xu, H., Jiang, N., Wang, D., Wang, L., Song, Y., Chen, Z., et al. Improving PMS oxidation of organic pollutants by single cobalt atom catalyst through hybrid radical and non-radical pathways. Applied Catalysis B: Environmental 263, 118350 (2020).

21. Li, X., Huang, X., Xi, S., Miao, S., Ding, J., Cai, W., et al. Single cobalt atoms anchored on porous Ndoped graphene with dual reaction sites for efficient Fenton-like catalysis. Journal of the American Chemical Society 140, 12469-12475 (2018).

22. Chu, C., Yang, J., Zhou, X., Huang, D., Qi, H., Weon, S., et al. Cobalt single atoms on tetrapyridomacrocyclic support for efficient peroxymonosulfate activation. Environmental Science \& Technology 55, 1242-1250 (2021).

23. Tian, H., Tian, H., Wang, S., Chen, S., Zhang, F., Song, L., et al. High-power lithium-selenium batteries enabled by atomic cobalt electrocatalyst in hollow carbon cathode. Nature Communications 11, 5025 (2020).

24. Li, H., Qian, J., Pan, B. N-coordinated Co containing porous carbon as catalyst with improved dispersity and stability to activate peroxymonosulfate for degradation of organic pollutants. Chemical 
Engineering Journal 403, 126395 (2021).

25. Gao, J., Yang, H., Huang, X., Hung, S., Cai, W., Jia, C., et al. Enabling direct $\mathrm{H}_{2} \mathrm{O}_{2}$ production in acidic media through rational design of transition metal single atom catalyst. Chem 6, 658-674 (2020).

26. Jung, E., Shin, H., Lee, B., Efremov, V., Lee, S., Lee, H., et al. Atomic-level tuning of Co-N-C catalyst for high-performance electrochemical $\mathrm{H}_{2} \mathrm{O}_{2}$ production. Nature Materials 19, 436-442 (2020).

27. Chen, Y., Gao, R., Ji, S., Li, H., Tang, K., Jiang, P., et al. Atomic-level modulation of electronic density at cobalt single-atom sites derived from metal-organic frameworks: Enhanced oxygen reduction performance. Angewandte Chemie International Edition 60, 3212-3221 (2021).

28. Li, F., Han, G., Bu, Y., Noh, H., Jeon, J., Shin, T., et al. Revealing isolated M-N $\mathrm{N}_{3} \mathrm{C} 1$ active sites for efficient collaborative oxygen reduction catalysis. Angewandte Chemie International Edition 59, 23678-23683 (2020).

29. Shang, H., Zhou, X., Dong, J., Li, A., Zhao, X., Liu, Q., et al. Engineering unsymmetrically coordinated Cu- $\mathrm{S}_{1} \mathrm{~N}_{3}$ single atom sites with enhanced oxygen reduction activity. Nature Communications 11, 3049 (2020).

30. Fan, K., Li, Z., Song, Y., Xie, W., Shao, M., Wei, M. Confinement synthesis based on layered double hydroxides: A new strategy to construct single-atom-containing integrated electrodes. Advanced Functional Materials 31, 2008064 (2021).

31. Rahm, M., Zeng, T., Hoffmann, R. Electronegativity seen as the ground-state average valence electron binding energy. Journal of the American Chemical Society 141, 342-351 (2019).

32. Wang, G., Nie, X., Ji, X., Quan, X., Chen, S., Wang, H., et al. Enhanced heterogeneous activation of peroxymonosulfate by $\mathrm{Co}$ and $\mathrm{N}$ codoped porous carbon for degradation of organic pollutants: the synergism between Co and N. Environmental Science: Nano 6, 399-410 (2019).

33. Dong, X., Ren, B., Zhang, X., Liu, X., Sun, Z., Li, C., et al. Diatomite supported hierarchical 2D CoNi $\mathrm{O}_{4}$ nanoribbons as highly efficient peroxymonosulfate catalyst for atrazine degradation. Applied Catalysis B: Environmental 272, 118971 (2020).

34. Jiao, L., Zhang, R., Wan, G., Yang, W., Wan, X., Zhou, H., et al. Nanocasting $\mathrm{SiO}_{2}$ into metal-organic frameworks imparts dual protection to high-loading Fe single-atom electrocatalysts. Nature Communications 11, 2831 (2020).

35. Jiao, L., Wan, G., Zhang, R., Zhou, H., Yu, S., Jiang, H. From Metal-organic frameworks to single-atom Fe implanted N-doped porous carbons: Efficient oxygen reduction in both alkaline and acidic media. Angewandte Chemie International Edition 57, 8525-8529 (2018). 
36. Zhang, Y., Zhang, R., Chen, S., Gao, H., Li, M., Song, X., et al. Diatomite-derived hierarchical porous crystalline-amorphous network for high-performance and sustainable Si anodes. Advanced Functional Materials 30, 2005956 (2020).

37. Li, J., Xu, J., Xie, Z., Gao, X., Zhou, J., Xiong, Y., et al. Diatomite-templated synthesis of freestanding 3D graphdiyne for energy storage and catalysis application. Advanced Materials 30, 1800548 (2018).

38. Chen, K., Li, C., Shi, L., Gao, T., Song, X., Bachmatiuk, A., et al. Growing three-dimensional biomorphic graphene powders using naturally abundant diatomite templates towards high solution processability. Nature Communications 7, 13440 (2016).

39. Zheng, Y., Fang, H., Wang, F., Huang, H., Yang, J., Zuo, G. Fabrication and characterization of mesoporous $\mathrm{Si} / \mathrm{SiC}$ derived from diatomite via magnesiothermic reduction. Journal of Solid State Chemistry 277, 654-657 (2019).

40. Shi, W., Liu, B., Deng, X., Li, J., Yang, Y. In-situ synthesis and properties of cordierite-bonded porous $\mathrm{SiC}$ membrane supports using diatomite as silicon source. Journal of the European Ceramic Society 36 , 3465-3472 (2016).

41. Song, L., Zhang, W., Wang, Y., Ge, X., Zou, L., Wang, H., et al. Tuning lithium-peroxide formation and decomposition routes with single-atom catalysts for lithium-oxygen batteries. Nature communications 11, 1-11 (2020).

42. Han, X., Ling, X., Yu, D., Xie, D., Li, L., Peng, S., et al. Atomically dispersed binary Co-Ni sites in nitrogen-doped hollow carbon nanocubes for reversible oxygen reduction and evolution. Advanced Materials 31, 1905622 (2019).

43. Fei, H., Dong, J., Wan, C., Zhao, Z., Xu, X., Lin, Z., et al. Microwave-assisted rapid synthesis of graphene-supported single atomic metals. Advanced Materials 30, 1802146 (2018).

44. Wang, P., Ren, Y., Wang, R., Zhang, P., Ding, M., Li, C., et al. Atomically dispersed cobalt catalyst anchored on nitrogen-doped carbon nanosheets for lithium-oxygen batteries. Nature Communications 11, 1-11 (2020).

45. Gnyla, J., Gubernat, A., Zych, Ł., Nocuń, M., Goral, Z., Lach, R. Influence of TMAH and NaOH on the stability of SiC aqueous suspensions. Ceramics International 46, 11208-11217 (2020).

46. Luo, H., Wang, L., Shang, S., Li, G., Lv, Y., Gao, S., et al. Cobalt nanoparticles-catalyzed widely applicable successive C-C bond cleavage in alcohols to access esters. Angewandte Chemie International Edition 59, 19268-19274 (2020).

47. Zhang, Q., Tan, X., Bedford, N., Han, Z., Thomsen, L., Smith, S., et al. Direct insights into the role of epoxy groups on cobalt sites for acidic $\mathrm{H}_{2} \mathrm{O}_{2}$ production. Nature Communications 11, 1-11 (2020). 
48. Gao, Y., Yang, C., Zhou, M., He, C., Cao, S., Long, Y., et al. Transition metal and metal-N $\mathrm{N}_{\mathrm{x}}$ codoped MOFderived Fenton-like catalysts: A comparative study on single atoms and nanoparticles. Smal/ 16, 2005060 (2020).

49. Marchetti, G., Minella, M., Maurino, V., Minero, C., Vione, D. Photochemical transformation of atrazine and formation of photointermediates under conditions relevant to sunlit surface waters: laboratory measures and modelling. Water Research 47, 6211-6222 (2013).

50. Zhu, S., Huang, X., Ma, F., Wang, L., Duan, X., Wang, S. Catalytic removal of aqueous contaminants on $\mathrm{N}$-doped graphitic biochars: Inherent roles of adsorption and nonradical mechanisms. Environmental Science \& Technology 52, 8649-8658 (2018).

51. Li, X., Wang, J., Duan, X., Li, Y., Fan, X., Zhang, G., et al. Fine-tuning radical/nonradical pathways on graphene by porous engineering and doping strategies. ACS Catalysis 11, 4848-4861 (2021).

\section{Figures}

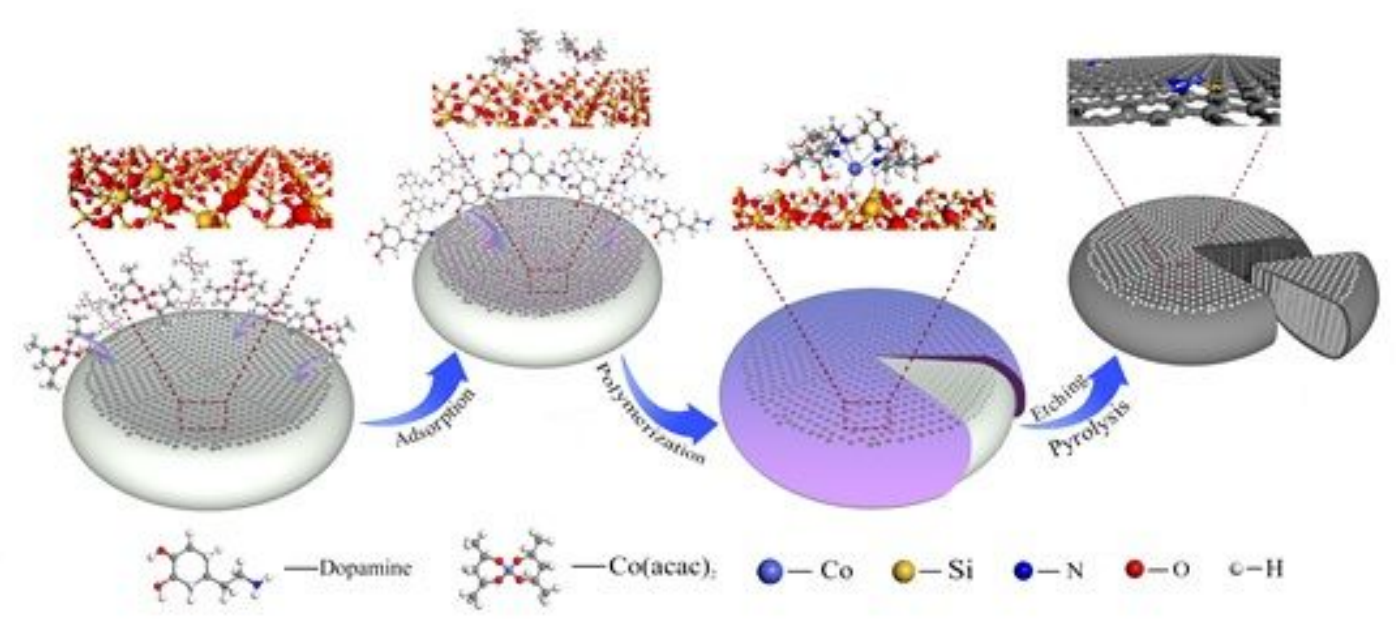

\section{Figure 1}

Schematic illustration of the synthesis route for p-CoSi1N3@D. 
a

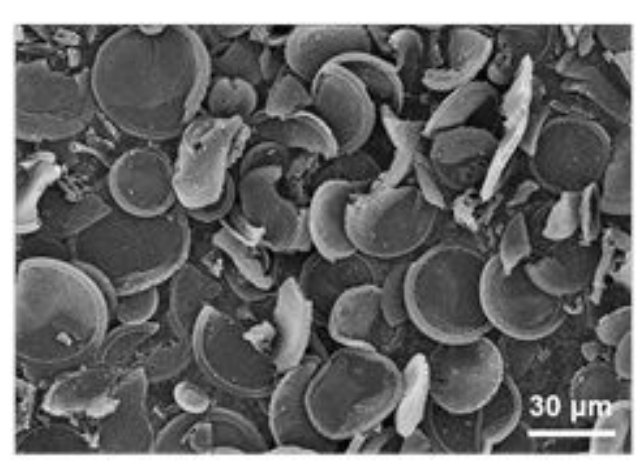

C

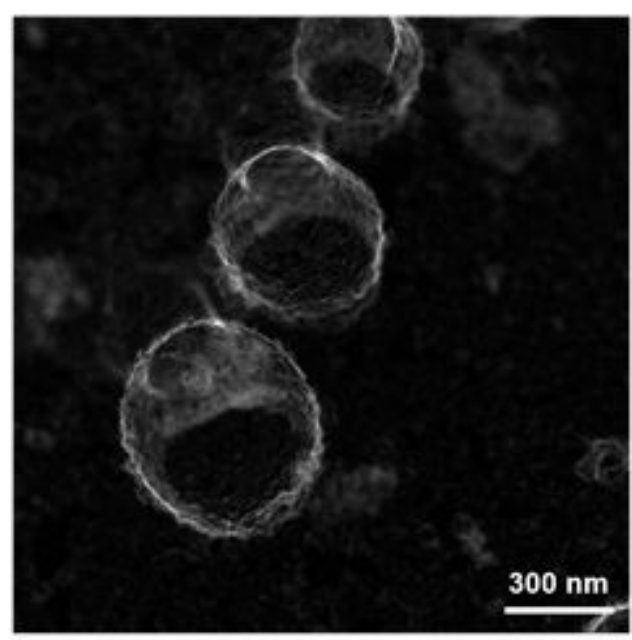

e

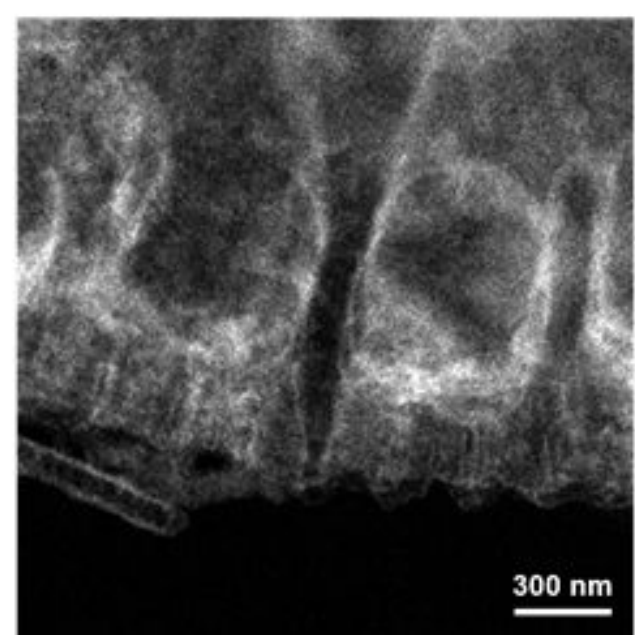

b

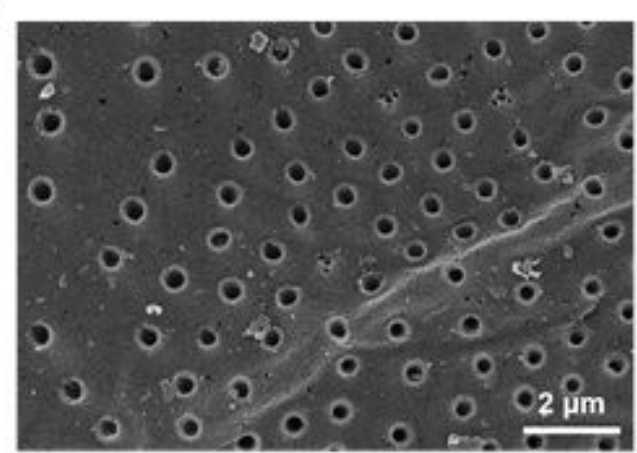

d

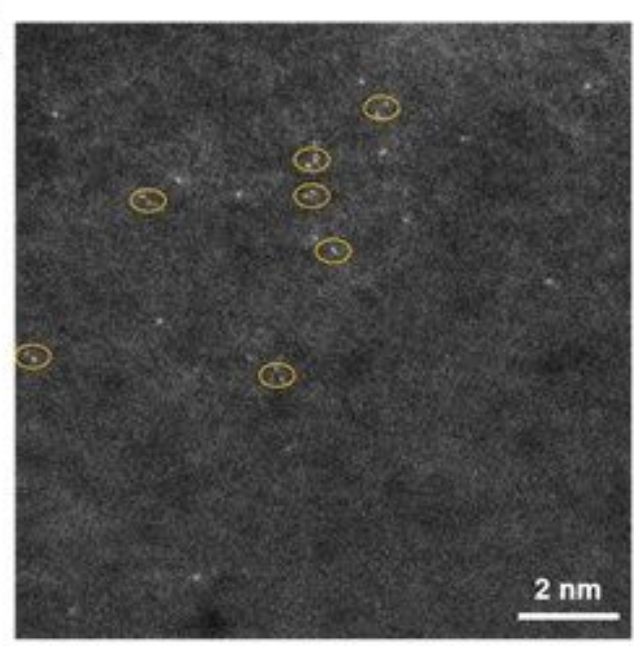

f
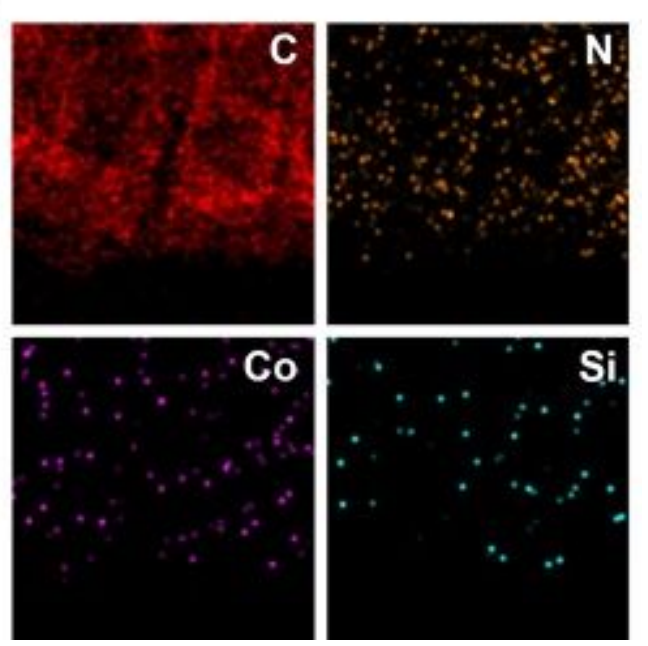

\section{Figure 2}

Structural characterizations of p-CoSi1N3@D. (a) and (b) SEM of p-CoSi1N3@D. (c) and (d) HADDFSTEM of p-CoSi1N3@D. (e) and (f) Corresponding EDX elemental mappings of p-CoSi1N3@D. 

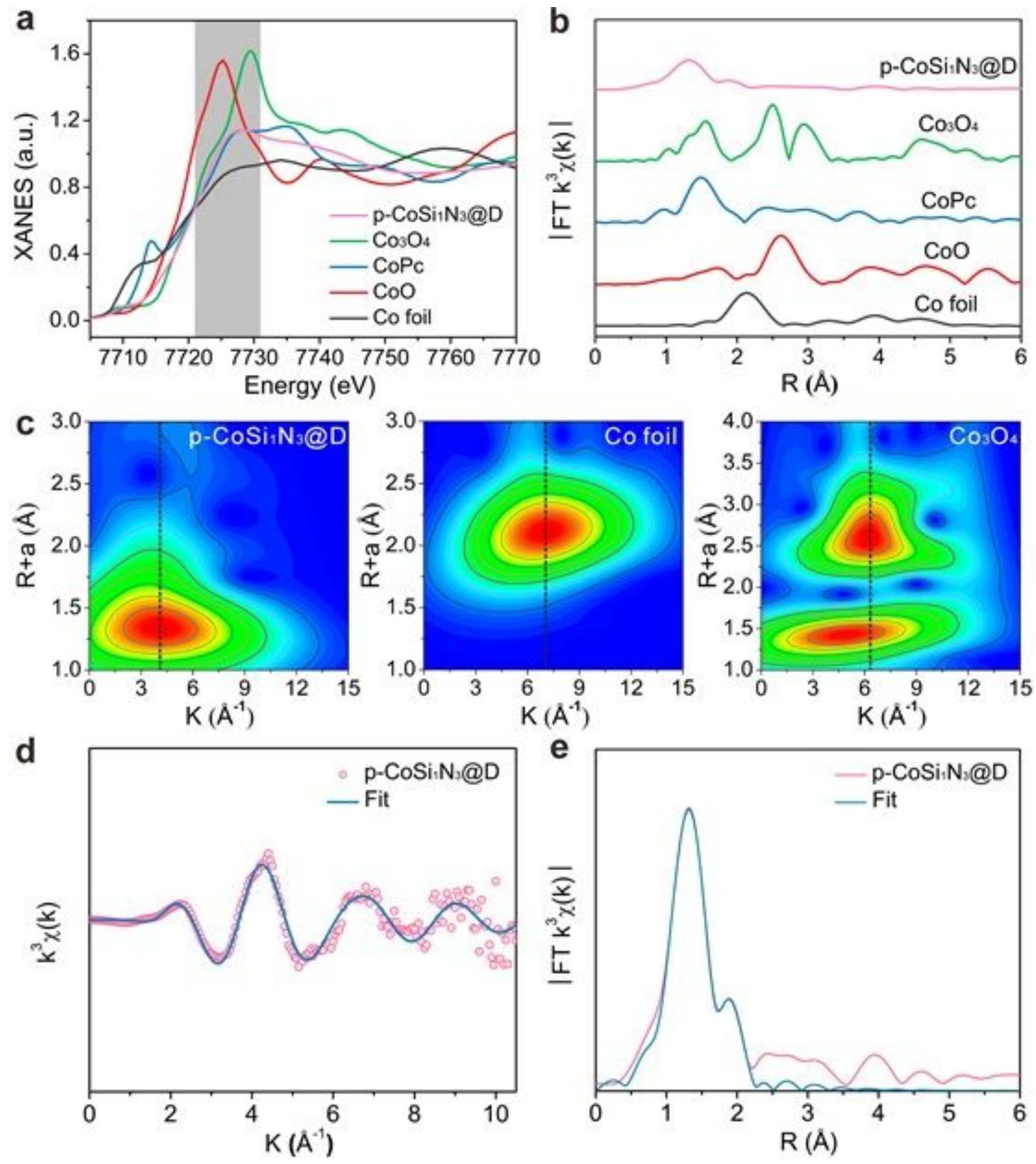

Figure 3

Identification for the spatial coordination configuration of cobalt single atom active sites in $\mathrm{p}$ CoSi1N3@D. (a) X-ray absorption near-edge structure (XANES) of p-CoSi1N3@D, CoPc, CoO, Co3O4 and Co foil (Co-K edge). (b) Fourier-transformed k3-weighted Extended X-ray absorption fine structure (EXAFS) (R space) of p-CoSi1N3@D, CoPc, CoO, Co3O4 and Co foil (Co-K edge). (c) Wavelet transform (WT) EXAFS (R space) of p-CoSi1N3@D, Co foil and Co304 (Co-K edge). Fitting curve EXAFS of p-CoSi1N3@D at (d) R space and (e) k space. 

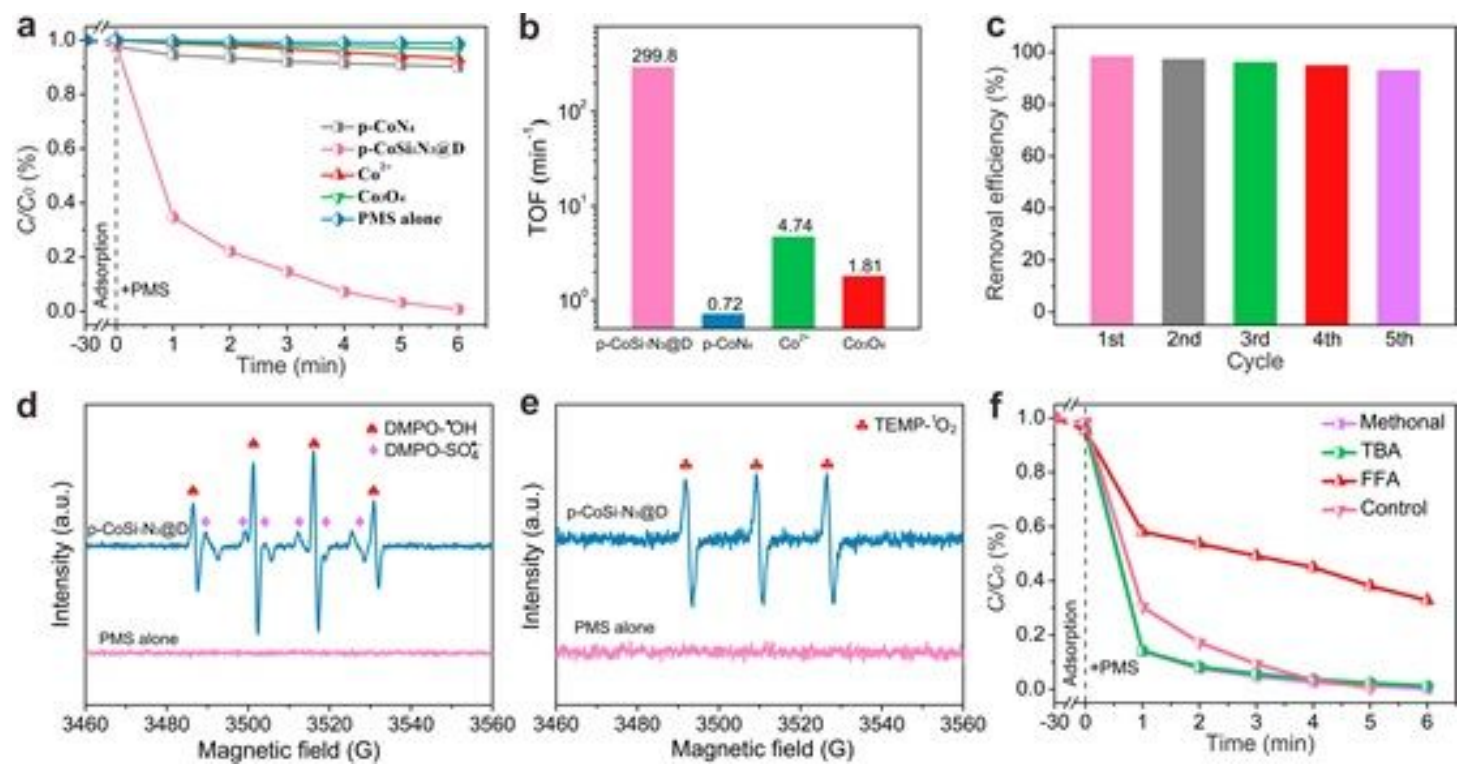

Figure 4

The catalytic performance and mechanism studies of p-CoSi1N3@D for PMS activation. (a) BPA degradation efficiency in different reaction systems. (b) TOF per Co atom basis for BPA removal calculated based on the initial BPA removal rate. (c) BPA degradation efficiency in five consecutive runs by p-CoSi1N3@D/PMS system (Experimental conditions: [BPA]0 $=20 \mathrm{mg} / \mathrm{L}$, [Catalyst]0 = $0.02 \mathrm{~g} / \mathrm{L}$, $[P M S] 0=0.4 \mathrm{mM}$, initial pH = 7.0). In situ EPR spectra under (d) p-CoSi1N3@D/PMS/DMPO and PMS/DMPO systems and (e) p-CoSi1N3@D/PMS/TEMP and PMS/TEMP systems. (f) Quenching effect on BPA degradation during PMS activation by different scavengers ([Methanol] $0=200 \mathrm{mM},[\mathrm{TBA}] 0=100$ $\mathrm{mM},[\mathrm{FFA}] 0=10 \mathrm{mM},[\mathrm{BPA}] 0=20 \mathrm{mg} / \mathrm{L} ;[$ Catalyst $] 0=0.02 \mathrm{~g} / \mathrm{L} ;[\mathrm{PMS}] 0=0.5 \mathrm{mM}$; initial $\mathrm{pH}=7.0)$. 
a

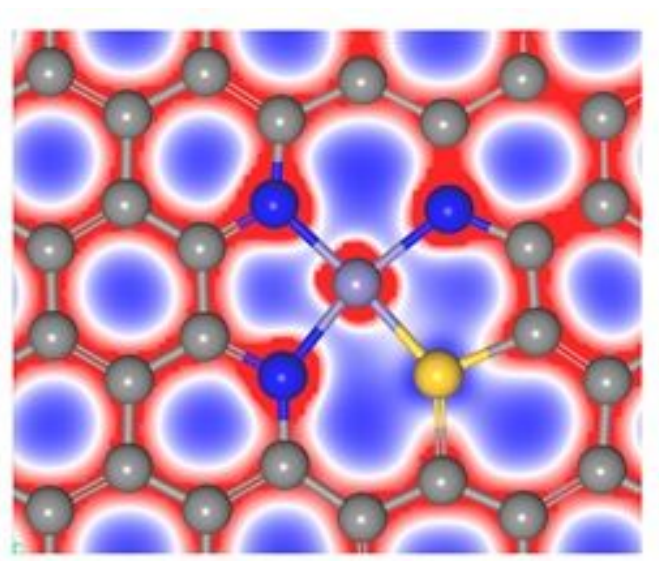

C

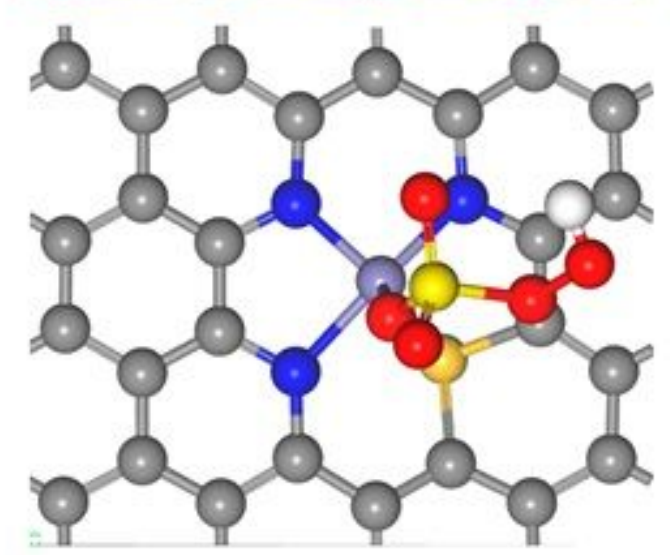

e

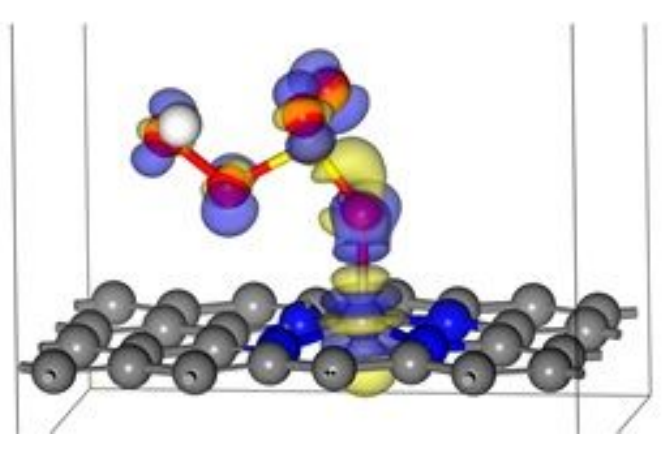

b

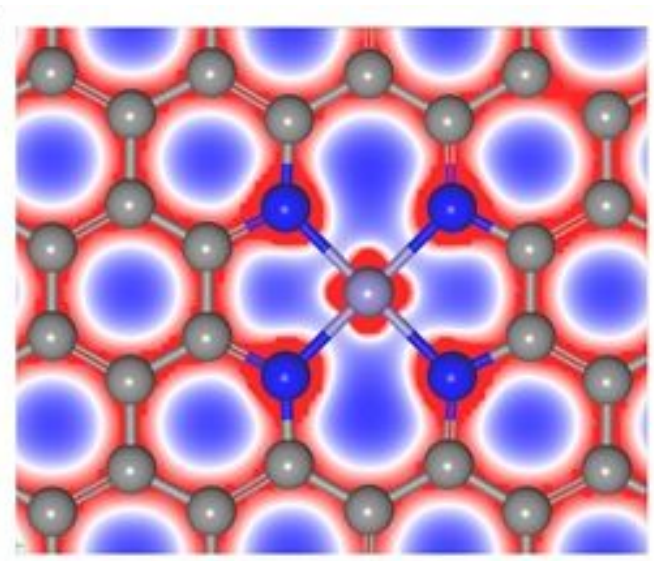

d

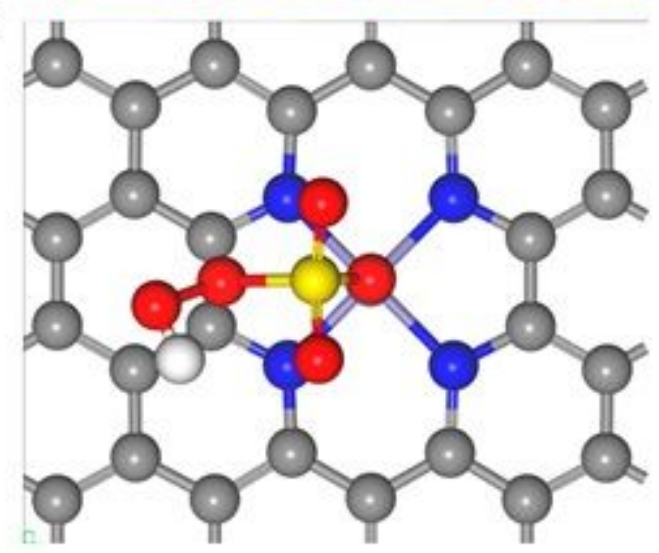

f

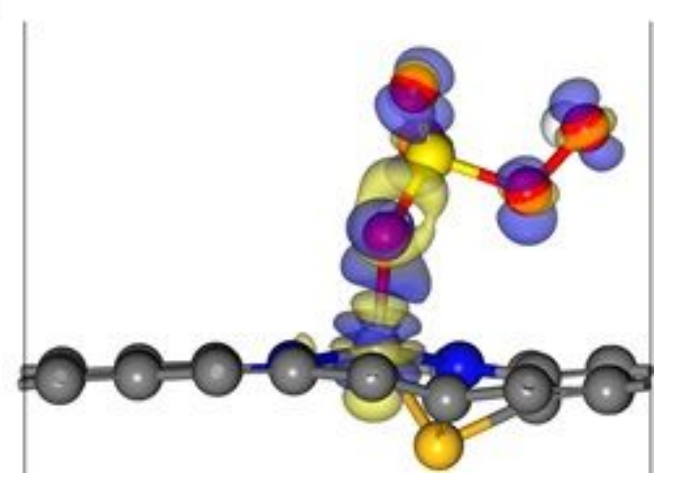

Figure 5

Theoretical calculations of p-CoSi1N3@D for enhanced PMS activation. 3D differential charge densities of (a) CoN4 and (b) CoSi1N3. The optimized adsorption structure of PMS molecules on (c) CoN4 and (d) CoSi1N3. 3D differential charge densities of PMS adsorbed on the Co for (e) CoN4 and (f) CoSi1N3.

\section{Supplementary Files}

This is a list of supplementary files associated with this preprint. Click to download.

- SupplementaryInformation.docx 